\title{
Hyperglycemia as a Risk Factor of Ischemic Stroke
}

\author{
Ziyan Zhang, Jingqi Yan and Honglian Shi*
}

Department of Pharmacology \& Toxicology, University of Kansas, Lawrence, Kansas, USA

\begin{abstract}
Diabetes is considered a major risk factor for stroke and is associated with worsened stroke outcomes. Here, we discuss and summarize the mechanisms that have been associated with the increased risk of stroke due to the hyperglycemia in diabetes mellitus. In diabetic stroke models, hyperglycemia exaggerates the following damaging processes: acidosis, accumulation of reactive oxygen species/reactive nitrogen, inflammation and mitochondrial dysfunction. Understanding the mechanism of diabetes acting as a stroke risk factor will definitely assist to reveal issues related to drug metabolism and toxicity in diabetic stroke. In addition, it is suggested that future studies may focus on the mechanisms mediating blood-brain barrier and astrocytes dysfunction under hyperglycemic stroke.
\end{abstract}

Keywords: Diabetes; Stroke; Hyperglycemia; BBB; Neuroprotection

\section{Introduction}

According to the World Health Organization, over 15 million people, equating to one in every 400 people, suffer a stroke worldwide a year [1]. Stroke is a leading cause of mortality after heart disease, and is responsible for about $9 \%$ of total deaths each year. It is also the single most common cause of long-term disability, with up to $40 \%$ of stroke patients not expected to recover independence [2]. A stroke can be due to ischemia caused by thrombosis or embolism or due to a hemorrhage. Ischemic stroke accounts for approximately $80-85 \%$ of all cases and is characterized by the disruption of cerebral blood flow and lack of oxygen to the affected area [3].

There is a substantial amount of clinical and experimental data showing the relationship between diabetes and stroke [4-6]. Diabetes is considered a risk factor particularly for ischemic stroke. Patients with diabetes are at 1.5-3 times the risk of stroke compared with the general population [7]. Diabetes also doubles the risk of stroke recurrence [8]. Furthermore, stroke outcomes are significantly worse among diabetic patients with increased mortality and neurological and functional disabilities [9]. Diabetes is a complex disease that extends beyond dysfunctional glucose regulation. People with diabetes generally have additional stroke risk factors, including hypertension, dyslipidemia, obesity, and atrial fibrillation. This review aims to discuss and summarize the mechanisms that have been associated with the increased risk of stroke due to the hyperglycemia in diabetes mellitus. According to a variety of findings in the literature, four main pathways, including acidosis, reactive oxygen species/reactive nitrogen species, inflammation, and mitochondrial dysfunction are involved in hyperglycemia-aggravated stroke.

\section{Acidosis}

Acidosis is considered to be a major contributor to neuronal damage in cerebral ischemia. Glucose is the sole energy substrate in the brain during both aerobic and anaerobic conditions. During anaerobic conditions such as in cerebral ischemia, glycolysis is the only process capable of producing significant amounts of ATP and lactate is the main product of glycolysis [10]. Hyperglycemia can worsen ischemic outcomes through aggravating acidosis in ischemic brain tissues. In 1977, Myers and Yamaguchi first reported that glucose administrated before cerebral ischemia significantly exacerbated the post-ischemic outcome [11]. Siesjo's lab conducted a series of experiments and reached the conclusion that infusion with glucose pre-ischemia led to the excessive amount of lactic acid. The accumulation of lactate resulted in a decrease of $\mathrm{pH}$ which is responsible for the excess damage in brains [12]. The exacerbation of post-ischemic brain injury is associated with enhancement of excitatory amino acid release, toxic metabolism of $\mathrm{NO}$, and hydroxyl radical formation due to the ischemia-acidosis [13].

Furthermore, neuroprotection provided by inhibiting glycolysis supports that acidosis plays important role in brain injury caused by ischemia and hyperglycemia. For example, pretreatment with 2-Deoxy-D-glucose (2DG), which can inhibit glycolysis, reduced mortality and morbidity in hyperglycemic rats under the condition of four-vessel occlusion [14]. 2DG also exerted a cytoprotective effect by preventing ischemia-induced hippocampal neuron damage in a gerbil transient forebrain ischemia model. By using ${ }^{1} \mathrm{H}$ NMR and MRI, Wei et al. concluded that inhibition of glucose metabolism by $2 \mathrm{DG}$ had a beneficial effect in reducing brain injury and minimizing the lactate production in brain during middle cerebral artery occlusion (MCAO)reperfusion in hyperglycemia rats [15].

However, it is still arguable whether lactate accumulation is directly detrimental to the ischemia brain. It has been reported that in an in vitro ischemia model, the combination of high glucose and acidosis, but not acidosis per se or the combination of lactate and acidosis exacerbated damage [16]. On the other hand, increased glucose concentrations also exacerbated ischemic injury in brain slices even when $\mathrm{pH}$ was tightly controlled [17]. Moreover, there is evidence demonstrating that pretreatment with high glucose $(20 \mathrm{mM})$ before hypoxia and mild acidosis has some protective effects against ischemia [10].

\section{Reactive Oxygen Species (ROS)}

During stroke, excessive production of ROS can lead to breakdown of the BBB and focal lesions. ROS is a group of natural by-products of oxygen metabolism including hydroxyl radical, superoxide and hydrogen peroxide. ROS have many detrimental effects, such as lipid

${ }^{*}$ Corresponding author: Honglian Shi, Ph.D. Associate Professor, Department of Pharmacology and Toxicology, University of Kansas, School of Pharmacy, 1251 Wescoe Hall Drive, Malott Hall 5044, Lawrence, KS 66045, USA, Tel: 1-785-8646193; Fax: 1-785-864-5219; E-mail: hshi@ku.edu

Received April 01, 2013; Accepted June 27, 2013; Published June 29, 2013

Citation: Zhang Z, Yan J, Shi H (2013) Hyperglycemia as a Risk Factor of Ischemic Stroke. J Drug Metab Toxicol 4: 153. doi:10.4172/2157-7609.1000153

Copyright: ( 2013 Zhang Z, et al. This is an open-access article distributed under the terms of the Creative Commons Attribution License, which permits unrestricted use, distribution, and reproduction in any medium, provided the original author and source are credited. 
peroxidation, protein denaturation, inactivation of enzymes, nucleic acid and DNA damage, release of $\mathrm{Ca}^{2+}$ from intracellular store, damage to the cytoskeletal structure and chemotaxis. All of these can lead to cell death and tissue destruction [18].

Several studies have demonstrated that hyperglycemia enhances the generation of ROSduring ischemia-reperfusion process $[19,20]$. Superoxide anion radical $\left(\mathrm{O}_{2}^{-}\right)$, the primary ROS, has been implicated in four major pathways leading to hyperglycemic complications: glucose-induced activation of protein kinase C (PKC) isoforms, increased formation of advanced glycation endproducts, increased glucose metabolism by aldose reductase pathway, and increased flux through the hexosamine pathway. Increased glycolysis results in higher NADH production and increased electron transfer through the electron transport chain in the mitochondrion. This results in the accumulation of a number of intermediates, which can transfer an electron to molecular oxygen to form $\mathrm{O}_{2}^{-}$In addition, PKCdependent activation of NADPH oxidase is also a significant source of $\mathrm{O}_{2}^{-}$in diabetes [21].

In diabetic rats, interestingly, MCAO for $2 \mathrm{~h}$ increased NADPH oxidase subunit expression to much higher levels than ischemiareperfusion (I/R) alone [22]. It was reported that activation of Rac and subsequently of the gp91 phox containing NADPH oxidase promoted cerebral ROS formation, which led to disruption of blood brain barrier [23]. Indeed, inhibition of NADPH oxidase was neuroprotective after I/R [24]. It was also reported that post-ischemic $\mathrm{O}_{2}^{-}$production and cell death were prevented or reduced by decreased glucose metabolism or inactivation of NADPH oxidase both in vitro and in vivo, identifying glucose as a requisite electron donor for reperfusion-induced neuronal $\mathrm{O}_{2}^{-}$production [25]. By using a novel electrochemical $\mathrm{O}_{2}^{-}$sensor, Tsuruta et al. demonstrated that following reperfusion from forebrain ischemia, hyperglycemia enhanced superoxide generation and lipid peroxidation in the brain [26].

ROS might play a central role in blood brain barrier (BBB) dysfunction during ischemia-reperfusion. They can change the vascular tone and therefore influence cerebral blood flow. Their vascular effects also include increasing platelet aggregability and endothelial cell permeability, altering reactivity to vasodilators, and leading to the formation of focal lesions in endothelial cell membranes [18]. Kamada et al. reported that hyperglycemia increased oxidative stress and matrix metalloproteinases-9 (MMP-9) activity, exacerbating BBB dysfunction after I/R [27]. Other studies showed that ROS induced degradation of the basement membrane and enhanced tyrosine phosphorylation of tight junctions by activating MMP-1,2,9 and decreasing tissue inhibitor of MMP (TIMP-1 and 2). Therefore, ROS production led to increased permeability and monocyte infiltration [28].

\section{Reactive Nitrogen Species (RNS)}

Reactive nitrogen species has also been reported to contribute to the exacerbated damage in stroke with diabetes $[29,30]$. Two important reactive nitrogen species are nitric oxide $(\mathrm{NO})$ and peroxynitrite (ONOO-). NO first known as endothelium-derived relaxing factor, which is induced during ischemia to increase vascular blood perfusion through increased expression of nitric oxide synthase (NOS) [31,32]. Since ischemia also increases the production of superoxide, the excessive nitric oxide forms a large amount of peroxynitrite [30]. Peroxynitrite induces damage in brain cells by directly causing protein S-nitrosylation, DNA fragmentation, and lipid peroxidation [33]. Moreover, peroxynitrite disrupts blood-brain barrier structure and increases the permeability [34]. Excessive peroxynitrite is also formed in diabetic vasculature and plays an important role in diabetes-induced vascular damage, both in experimental models and in humans $[35,36]$. High glucose levels result in increased nitric oxide and superoxide production, leading to peroxynitrite formation [37-39]. Given both ischemia and hyperglycemia induces the formation of peroxynitrite, the peroxynitrite level in diabetic stroke was much higher than non-diabetic stroke [30]. The immunoreactivity of nitrotyrosine, products of proteins oxidation by peroxynitrite, is more prominent in hyperglycemic stroke [29]. Reducing the peroxynitrite formation in hyperglycemic MCAO rat brains with NOS inhibitor, L-nitroarginine methyl ester (L-NAME), could decrease the infracted region to the levels observed in normoglycemic rats [13]. In this way, peroxynitrite may serve as one of the mediators for the severe brain damage in diabetic stroke.

\section{Inflammation}

Accumulating evidence suggests that cerebral ischemia elicits inflammation. During ischemia, the circulating cells including neutrophils, monocytes/macrophages and resident cells including microglia, astrocytes, and endothelial cells secrete inflammatory cytokines in the damaged areas [40]. Inflammatory cytokines such as tumor necrosis factor-alpha (TNFa) and interleukin-1beta (IL$1 \beta$ ) cause cellular adhesion molecule expression on endothelial cells which increases polymorphonuclear (PMN) leukocytes and other inflammatory cells to adhere to the endotheRal cells [41]. These cell-bound leukocytes then release MMPs, which participate in the breakdown of neurovascular matrix with consequent BBB disruption and edema. Furthermore, adhesion of leukocytes induces burst of production of ROS that contribute to secondary injury of BBB [42].

Many studies have provided evidence for a linkage between diabetes and inflammation. Nuclear factor kappa B (NF- $\kappa \mathrm{B})$ controls the induction of many inflammatory genes. During hyperglycemia, $\mathrm{NF}-\kappa \mathrm{B}$ is rapidly and dramatically activated in vascular cells and results in a subsequent increase in leukocytes adhesion and transcription of pro-inflammatory cytokines [6]. Glucose intake also causes an increase in early growth response-1 (Egr-1) that modulates the transcription of tissue factor (TF). Aljada et al. reported that glucose-induced increase in TF worsened ischemic damage by promoting coagulation in local capillaries [43]. Kim et al. found that chronic high glucose exposure of leukocytes increased their binding to human aortic endothelial cells [44]. Panes et al. observed diabetic hyperglycemia was associated with exaggerated leukocytes-endothelial cell adhesion and albumin leakage in response to $I / R$, setting a stage for an increased inflammatory response [45].

There are several reports suggesting that inflammatory responses might mediate hyperglycemia-aggravated brain damage induce by $\mathrm{I} / \mathrm{R}$. At post-translational levels, IL- $1 \beta$ and COX-2 expressions were significantly higher following hyperglycemic ischemia than hyperglycemic shams [46]. By using myeloperoxidase immunohistochemistry, Lin et al. demonstrated that hyperglycemia triggered early, massive deposition of neutrophils in the post-ischemic brain, which might exacerbate injury [47]. This increased adhesion of leukocytes to the endothelial cells was largely due to the increased expression of cell adhesion molecules on endothelial cells. It was reported that the number of intercellular adhesion molecule 1 (ICAM1)-stained microvessels in the cortex was markedly increased at 3 days following $\mathrm{I} / \mathrm{R}$ in diabetic, but not non-diabetic rats. Western blott showed that IL- $1 \beta$ was increased after 3 days of reperfusion in diabetic rats, suggesting that IL- $1 \beta$ might mediate ICAM- 1 expression in 
diabetic animals [48]. Tsuruta et al. demonstrated that hyperglycemia enhanced the expression of high-mobility group box 1 (HMGB1) in the brain cytoplasm. HMGB1 is reported to be released early from neurons following ischemic injury. It acts as an upstream inflammatory signal by inducing other pro-inflammatory cytokines expression [26]. Therefore, the elevation of HMGB1 during hyperglycemic ischemia may contribute to inflammatory response.

\section{Mitochondrial Dysfunction}

Another mechanism involving worsened outcomes of stroke is diabetes-induced mitochondrial dysfunction. Moreira et al. demonstrated that mitochondria isolated from the brains of STZinduced diabetic rats possessed a lower content of antioxidant defenses (CoQ) and higher oxidative stress than control [49]. The activities of mitochondrial enzymes, NADH dehydrogenase, succinate dehydrogenase and cytochrome oxidase, were decreased in diabetic rat brains [50]. Increased cytochrome $\mathrm{C}$ and active caspase- 3 levels were also observed in cytosol of diabetic rat brain cells [50]. The impaired mitochondrial functions by diabetes make the neurons more vulnerable to stroke because ischemia itself also induces mitochondrial dysfunction through oxidative stress and glutamate release [51,52], increases mitochondrial ROS production, reduces respiratory complex activities, and causes the release of cytochrome $\mathrm{C}$, all of which serve as important pathways for stroke-induced neuron death [53,54]. It has been reported that severe neuron death in diabetic rats after MCAO was associated with mitochondrial dysfunction and increased cytochrome C release [55].

Besides neuronal cells, diabetes was reported to impair mitochondrial functions in brain endothelial cells [56]. In diabetes, endothelial cells apoptosis and dysfunction was induced by increased mitochondrial ROS generation, impaired mitochondrial energy production, and release of apoptotic factors [57-60]. It was suggested that vasculatures needed to respond quickly to increased blood perfusion and attenuate the inflammatory reactions to protect brain from ischemic damage [61]. Normally, endothelial cells secrete vasodilator molecules to modulate vascular tone and increase blood supply during ischemia $[61,62]$. Nitric oxide, one of the endothelial vasodilators, can suppress inflammatory reactions by preventing leukocyte adhesion and reducing the expression of pro-inflammatory factors [63]. Therefore, both endothelial cell death and dysfunction limit their capacity of responding to ischemic damage. However, more research is needed to understand the mechanisms of diabetesinduced mitochondrial dysfunction-mediated vascular dysfunction and exacerbated outcomes of stroke.

\section{Other Related Factors}

There are other mediators which are not tightly related to the above mechanisms but regulate brain damage during hyperglycemic ischemia. Hypoxia inducible faction 1 (HIF-1) is a key factor in mediating a series of genes that induce erythropoiesis, apoptosis, antiapoptosis, necrosis, and angiogenesis during ischemic brain injury. In tissues from diabetic animals and patients, HIF-1 $\alpha$ functional activity decreased due to impaired HIF-1a binding to the coactivator p300. In setting of hypoxia, vascular endothelial cell growth factor (VEGF) is directly controlled by HIF-1. VEGF production in response to hypoxia was decreased in diabetic tissues [64]. In acute hyperglycemia-induced hemorrhagic transformation in a rat model of focal cerebral ischemia, the inhibition of HIF-1 $\alpha$ and its downstream genes attenuated hemorrhagic transformation (extravasation of blood cellular elements), reduced cerebral infarction and ameliorated neurological deficits [65].
Rho and Rho-associated kinase (ROCK) play pivotal roles in the pathogenesis in stroke. ROCK activation is considered to increase the risk of cerebral ischemia and worsen the ischemic tissue outcome and functional recovery. Rho/ROCK activity was increased systemically as well as in cerebral arteries in both Type 1 and Type 2 diabetic animal models [66]. As a result, Rho is considered to be involved in the increased risk of stroke in diabetes.

\section{Future Direction}

Many studies suggest that hyperglycemia worsens the outcome and increases the risk of ischemia. The process is complex. The possible mechanisms are interdependent, interactive, and possibly modulate each other. However, it seems that ROS play a central role in all the possible mechanisms modulating increased brain damage. For example, acidosis can enhance formation of free radicals. Hyperglycemiainduced oxidative stress and generation of ROS is also responsible for the activation of many inflammatory cytokines while the subsequent inflammation response will in turn generate large amount of ROS.

Though many studies focused on diabetes-induced vascular complications, there are few studies specifically focused on the mechanisms of BBB dysfunction under hyperglycemic stroke. Further research can be focused on the mechanism of BBB's damage.

It is known that during the development of diabetes a number of biochemical and mechanical factors converge on the endothelium, resulting in endothelial dysfunction and vascular inflammation. This provides a basis for the vascular disease seen in diabetes [67]. It is also reported that during inflammation, HIF-1 levels were upregulated in neutrophils and macrophage [68]. However, there is no research on HIF-1's effects on cerebral vascular inflammation in diabetes. Besides, HIF-1 plays an important role in modulating BBB permeability during stroke. The increasing permeability of $\mathrm{BBB}$ in stroke can provide more oxygen and nutrients to neuronal cells, however, it can also cause brain edema due to leakage of blood components [69]. We postulate that diabetic inflammation may regulate activity of HIF-1 in brain endothelial cells, leading to aggravation of brain injury in stroke.

Astrocytes are glial cells that envelop $>99 \%$ of the BBB endothelium. Interaction of astrocytes with endothelial cells greatly enhances endothelial cell tight junctions and maintains BBB tightness and function [70]. Hypoxic exposure induced a higher level of VEGF releasing from astrocytes, which provides protective effect to neighboring cells [71]. High glucose can potentiate the increase of hemichannel activity and impairment of gap junctional communication among astrocytes under hypoxia. Thus, hyperglycemia resulted in astrocytes dysfunction and death [72]. It is highly possible that in hyperglycemic stroke, dysfunction of astrocytes is a major factor resulting in BBB damage.

So far numerous neuroprotective drugs have been investigated in animal models of ischemic stroke, many of which have achieved general success in preclinical studies across disparate animal models [73] However, of the more than 100 neuroprotective agents that reached randomized clinical trials in focal ischemic stroke, none has proven unequivocally efficacious, despite success seen in preceding animal studies [74]. The translational disappointment of neuroprotective agents likely arise from a combination of factors including poor choice of the agents and time of administration, the molecular mechanism targeted, and difference between animal models and human pathological conditions [75].

In summary, hyperglycemia may increase stroke occurrence and exacerbate stroke outcome through modulating acidosis, free radical 
Citation: Zhang Z, Yan J, Shi H (2013) Hyperglycemia as a Risk Factor of Ischemic Stroke. J Drug Metab Toxicol 4: 153. doi:10.4172/21577609.1000153

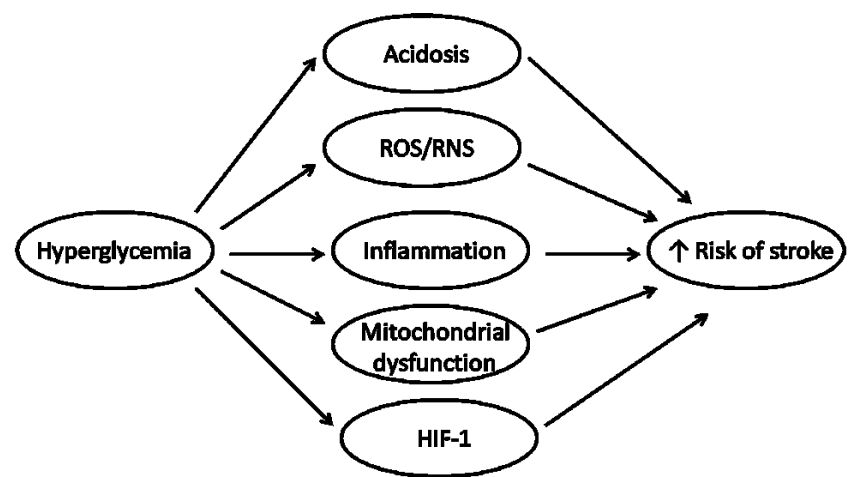

Scheme 1: Possible mechanism of hyperglycemia-mediated increased stroke occurrence and exacerbated stroke outcome.

generation, mediators of inflammation, mitochondrial function and other factors such as HIF-1 (Scheme 1). All the factors may have impact on drug metabolism and toxicity in diabetic stroke.

\section{Acknowledgements}

This research was supported in part by a grant from the National Institutes of Health (R01NS058807) and a Kansas University Center for Research startup fund.

\section{References}

1. Weir NU, Dennis MS (1997) Meeting the challenge of stroke. Scott Med J 42: 145-147.

2. Caplan LR, Hon FK (2004) Clinical diagnosis of patients with cerebrovascular disease. Prim Care 31: 95-109.

3. Lyden PD, Zivin JA (1993) Hemorrhagic transformation after cerebral ischemia: mechanisms and incidence. Cerebrovasc Brain Metab Rev 5: 1-16.

4. Capes SE, Hunt D, Malmberg K, Pathak P, Gerstein HC (2001) Stress hyperglycemia and prognosis of stroke in nondiabetic and diabetic patients: a systematic overview. Stroke 32: 2426-2432.

5. Gray CS, Hildreth AJ, Sandercock PA, O'Connell JE, Johnston DE, et al. (2007) Glucose-potassium-insulin infusions in the management of post-stroke hyperglycaemia: the UK Glucose Insulin in Stroke Trial (GIST-UK). Lancet Neurol 6: 397-406.

6. Martini SR, Kent TA (2007) Hyperglycemia in acute ischemic stroke: a vascular perspective. J Cereb Blood Flow Metab 27: 435-451.

7. Sander D, Kearney MT (2009) Reducing the risk of stroke in type 2 diabetes: pathophysiological and therapeutic perspectives. J Neurol 256: 1603-1619.

8. Kissela BM, Khoury J, Kleindorfer D, Woo D, Schneider A, et al. (2005) Epidemiology of ischemic stroke in patients with diabetes: the greater Cincinnati/Northern Kentucky Stroke Study. Diabetes Care 28: 355-359.

9. Idris I, Thomson GA, Sharma JC (2006) Diabetes mellitus and stroke. Int J Clin Pract 60: 48-56.

10. Schurr A (2001) Glucose and the ischemic brain: a sour grape or a sweet treat? Curr Opin Clin Nutr Metab Care 4: 287-292.

11. Myers RE, Yamaguchi S (1977) Nervous system effects of cardiac arrest in monkeys. Preservation of vision. Arch Neurol 34: 65-74.

12. Siesjo BK (1988) Acidosis and ischemic brain damage. Neurochem Pathol 9 : 31-88.

13. Wei J, Quast MJ (1998) Effect of nitric oxide synthase inhibitor on a hyperglycemic rat model of reversible focal ischemia: detection of excitatory amino acids release and hydroxyl radical formation. Brain Res 791: 146-156.

14. Combs DJ, Reuland DS, Martin DB, Zelenock GB, D'Alecy LG (1986) Glycolytic inhibition by 2-deoxyglucose reduces hyperglycemia-associated mortality and morbidity in the ischemic rat. Stroke 17: 989-994.

15. Wei J, Cohen DM, Quast MJ (2003) Effects of 2-deoxy-d-glucose on focal cerebral ischemia in hyperglycemic rats. J Cereb Blood Flow Metab 23: 556564.
16. Cronberg T, Rytter A, Asztely F, Soder A, Wieloch T (2004) Glucose but not lactate in combination with acidosis aggravates ischemic neuronal death in vitro. Stroke 35: 753-757

17. Newell DW, Barth A, Papermaster V, Malouf AT (1995) Glutamate and nonglutamate receptor mediated toxicity caused by oxygen and glucose deprivation in organotypic hippocampal cultures. J Neurosci 15: 7702-7711.

18. Allen CL, Bayraktutan U (2009) Oxidative stress and its role in the pathogenesis of ischaemic stroke. Int J Stroke 4: 461-470.

19. Wei J, Huang NC, Quast MJ (1997) Hydroxyl radical formation in hyperglycemic rats during middle cerebral artery occlusion/reperfusion. Free Radic Biol Med 23: $986-995$

20. Li PA, Liu GJ, He QP, Floyd RA, Siesjo BK (1999) Production of hydroxyl free radical by brain tissues in hyperglycemic rats subjected to transient forebrain ischemia. Free Radic Biol Med 27: 1033-1040.

21. Brownlee M (2001) Biochemistry and molecular cell biology of diabetic complications. Nature 414: 813-820

22. Kusaka I, Kusaka G, Zhou C, Ishikawa M, Nanda A, et al. (2004) Role of AT receptors and $\mathrm{NAD}(\mathrm{P}) \mathrm{H}$ oxidase in diabetes-aggravated ischemic brain injury. Am J Physiol Heart Circ Physiol 286: H2442-H2451.

23. Kahles T, Luedike P, Endres M, Galla HJ, Steinmetz H, et al. (2007) NADPH oxidase plays a central role in blood-brain barrier damage in experimental stroke. Stroke 38:3000-3006.

24. Chen H, Song YS, Chan PH (2009) Inhibition of NADPH oxidase is neuroprotective after ischemia-reperfusion. J Cereb Blood Flow Metab 29 $1262-1272$.

25. Suh SW, Shin BS, Ma H, Van Hoecke M, Brennan AM, et al. (2008) Glucose and NADPH oxidase drive neuronal superoxide formation in stroke. Ann Neurol 64: 654-663.

26. Tsuruta R, Fujita M, Ono T, Koda Y, Koga Y, et al. (2010) Hyperglycemia enhances excessive superoxide anion radical generation, oxidative stress, early inflammation, and endothelial injury in forebrain ischemia/reperfusion rats. Brain Res 1309: 155-163.

27. Kamada H, Yu F, Nito C, Chan PH (2007) Influence of hyperglycemia on oxidative stress and matrix metalloproteinase-9 activation after focal cerebra ischemia/reperfusion in rats: relation to blood-brain barrier dysfunction. Stroke 38: 1044-1049.

28. Haorah J, Ramirez SH, Schall K, Smith D, Pandya R, et al. (2007) Oxidative stress activates protein tyrosine kinase and matrix metalloproteinases leading to blood-brain barrier dysfunction. J Neurochem 101: 566-576.

29. Ste-Marie L, Hazell AS, Bemeur C, Butterworth R, Montgomery J (2001) Immunohistochemical detection of inducible nitric oxide synthase, nitrotyrosine and manganese superoxide dismutase following hyperglycemic focal cerebral ischemia. Brain Res 918: 10-19.

30. Palomares SM, Gardner-Morse I, Sweet JG, Cipolla MJ (2012) Peroxynitrite decomposition with FeTMPyP improves plasma-induced vascular dysfunction and infarction during mild but not severe hyperglycemic stroke. J Cereb Blood Flow Metab 32: 1035-1045

31. Zhang ZG, Chopp M, Zaloga C, Pollock JS, Forstermann U (1993) Cerebra endothelial nitric oxide synthase expression after focal cerebral ischemia in rats. Stroke 24: 2016-2021.

32. Zhang ZG, Chopp M, Gautam S, Zaloga C, Zhang RL, et al. (1994) Upregulation of neuronal nitric oxide synthase and mRNA, and selective sparing of nitric oxide synthase-containing neurons after focal cerebral ischemia in rat. Brain Res 654: 85-95.

33. BÃ@meur C, Ste-Marie L, Montgomery J (2007) Increased oxidative stress during hyperglycemic cerebral ischemia. Neurochem Int 50: 890-904.

34. Tan KH, Harrington S, Purcell WM, Hurst RD (2004) Peroxynitrite mediates nitric oxide-induced blood-brain barrier damage. Neurochem Res 29: 579-587.

35. Pacher P, Szabo C (2008) Role of the peroxynitrite-poly(ADP-ribose) polymerase pathway in human disease. Am J Pathol 173: 2-13

36. Molnar A, Toth A, Bagi Z, Papp Z, Edes I, et al. (2006) Activation of the poly(ADP-ribose) polymerase pathway in human heart failure. Mol Med 12 : 143-152.

37. Hoshiyama M, Li B, Yao J, Harada T, Morioka T, et al. (2003) Effect of high 
Citation: Zhang Z, Yan J, Shi H (2013) Hyperglycemia as a Risk Factor of Ischemic Stroke. J Drug Metab Toxicol 4: 153. doi:10.4172/21577609.1000153

glucose on nitric oxide production and endothelial nitric oxide synthase protein expression in human glomerular endothelial cells. Nephron Ex Nephr 95:e6268.

38. Savino A, Pelliccia P, Schiavone C, Primavera A, Tumini S, et al. (2006) Serum and urinary nitrites and nitrates and Doppler sonography in children with diabetes. Diabetes Care 29: 2676-2681.

39. Son SM (2012) Reactive oxygen and nitrogen species in pathogenesis of vascular complications of diabetes. Diabetes Metab J 36: 190-198.

40. Feuerstein GZ, Wang X, Barone FC (1998) The role of cytokines in the neuropathology of stroke and neurotrauma. Neuroimmunomodulation 5: 143 159.

41. Albelda SM, Smith CW, Ward PA (1994) Adhesion molecules and inflammatory injury. FASEB J 8: 504-512.

42. Clark RK, Lee EV, Fish CJ, White RF, Price WJ, et al. (1993) Development of tissue damage, inflammation and resolution following stroke: an immunohistochemical and quantitative planimetric study. Brain Res Bull 31: 565-572.

43. Aljada A, Ghanim H, Mohanty P, Syed T, Bandyopadhyay A, et al. (2004) Glucose intake induces an increase in activator protein 1 and early growth response 1 binding activities, in the expression of tissue factor and matrix metalloproteinase in mononuclear cells, and in plasma tissue factor and matrix metalloproteinase concentrations. Am J Clin Nutr 80: 51-57.

44. Kim JA, Berliner JA, Natarajan RD, Nadler JL (1994) Evidence that glucose increases monocyte binding to human aortic endothelial cells. Diabetes 43 : $1103-1107$.

45. Panes J, Kurose I, Rodriguez-Vaca D, Anderson DC, Miyasaka M, et al. (1996) Diabetes exacerbates inflammatory responses to ischemia-reperfusion. Circulation 93: 161-167.

46. Bemeur C, Ste-Marie L, Desjardins P, Vachon L, Butterworth RF, et al (2005) Dehydroascorbic acid normalizes several markers of oxidative stress and inflammation in acute hyperglycemic focal cerebral ischemia in the rat. Neurochem Int 46: 399-407.

47. Lin B, Ginsberg MD, Busto R, Li L (2000) Hyperglycemia triggers massive neutrophil deposition in brain following transient ischemia in rats. Neurosci Lett 278: $1-4$

48. Ding C, He Q, Li PA (2005) Diabetes increases expression of ICAM after a brief period of cerebral ischemia. J Neuroimmunol 161: 61-67.

49. Moreira PI, Santos MS, Sena C, Seica R, Oliveira CR (2005) Insulin protects against amyloid beta-peptide toxicity in brain mitochondria of diabetic rats. Neurobiol Dis 18: 628-637.

50. Kamboj SS, Sandhir R (2011) Protective effect of N-acetylcysteine supplementation on mitochondrial oxidative stress and mitochondrial enzymes in cerebral cortex of streptozotocin-treated diabetic rats. Mitochondrion 11 : 214-222.

51. Schinder AF, Olson EC, Spitzer NC, Montal M (1996) Mitochondrial dysfunction is a primary event in glutamate neurotoxicity. J Neurosci 16: 6125-6133.

52. Chrissobolis S, Miller AA, Drummond GR, Kemp-Harper BK, Sobey CG (2011) Oxidative stress and endothelial dysfunction in cerebrovascular disease. Front Biosci 16: 1733-1745.

53. Sims NR, Anderson MF (2002) Mitochondrial contributions to tissue damage in stroke. Neurochem Intl 40: 511-526.

54. Sugiyama S, Miyazaki Y, Kotaka K, Ozawa T (1982) On the mechanism of ischemia-induced mitochondrial dysfunction. Japn Circ J 46: 296-302.

55. Muranyi M, Fujioka M, He Q, Han A, Yong G, et al. (2003) Diabetes activates cell death pathway after transient focal cerebral ischemia. Diabetes 52: 481-
56. Fatehi-Hassanabad Z, Chan CB, Furman BL (2010) Reactive oxygen species and endothelial function in diabetes. Eur J Pharmacol 636: 8-17.

57. Fujisawa K, Nishikawa T, Kukidome D, Imoto K, Yamashiro T, et al. (2009) TZDs reduce mitochondrial ROS production and enhance mitochondrial biogenesis. Biochem Biophys Res Commun 379: 43-48.

58. Pangare M, Makino A (2012) Mitochondrial function in vascular endothelial cel in diabetes. J Smooth Muscle Res 48: 1-26

59. Cheng X, Siow RC, Mann GE (2011) Impaired redox signaling and antioxidant gene expression in endothelial cells in diabetes: a role for mitochondria and the nuclear factor-E2-related factor 2-Kelch-like ECH-associated protein 1 defense pathway. Antioxid Redox Signal 14: 469-487.

60. Huang QR, Li Q, Chen YH, Li L, Liu LL, et al. (2010) Involvement of anion exchanger-2 in apoptosis of endothelial cells induced by high glucose through an mPTP-ROS-Caspase-3 dependent pathway. Apoptosis 15: 693-704.

61. Widlansky ME, Gokce N, Keaney JF Jr, Vita JA (2003) The clinical implications of endothelial dysfunction. J Am Coll Cardiol 42: 1149-1160.

62. Toda N, Ayajiki K, Okamura T (2009) Cerebral blood flow regulation by nitric oxide: recent advances. Pharmacol Rev 61: 62-97.

63. De Caterina R, Libby P, Peng HB, Thannickal VJ, Rajavashisth TB, et al. (1995) Nitric oxide decreases cytokine-induced endothelial activation. Nitric oxide selectively reduces endothelial expression of adhesion molecules and proinflammatory cytokines. J Clinic Invest 96: 60-68.

64. Thangarajah H, Yao D, Chang El, Shi Y, Jazayeri L, et al. (2009) The molecular basis for impaired hypoxia-induced VEGF expression in diabetic tissues. Proc Natl Acad Sci U S A 106: 13505-13510.

65. Chen C, Ostrowski RP, Zhou C, Tang J, Zhang JH (2010) Suppression of hypoxia-inducible factor-1alpha and its downstream genes reduces acute hyperglycemia-enhanced hemorrhagic transformation in a rat model of cerebral ischemia. J Neurosci Res 88: 2046-2055.

66. Shin HK, Salomone S, Ayata C (2008) Targeting cerebrovascular Rho-kinase in stroke. Expert Opin Ther Targets 12: 1547-1564.

67. Hartge MM, Unger T, Kintscher U (2007) The endothelium and vascular inflammation in diabetes. Diab Vasc Dis Res 4: 84-88.

68. Dehne N, BrÃ $1 / 4$ ne B (2009) HIF-1 in the inflammatory microenvironment. Exp Cell Res 315: 1791-1797.

69. Ke Q, Costa M (2006) Hypoxia-inducible factor-1 (HIF-1). Mol Pharmacol 70: 1469-1480.

70. Persidsky Y, Ramirez SH, Haorah J, Kanmogne GD (2006) Blood-brain barrier: structural components and function under physiologic and pathologic conditions. J Neuroimmune Pharmacol 1: 223-236.

71. Schmid-Brunclik N, Burgi-Taboada C, Antoniou X, Gassmann M, Ogunshola OO (2008) Astrocyte responses to injury: VEGF simultaneously modulates cel death and proliferation. Am J Physiol Regul Integr Comp Physiol 295: R864 873.

72. Orellana JA, HernÃ indez DE, Ezan P, Velarde V, Bennett MV, et al. (2010) Hypoxia in high glucose followed by reoxygenation in normal glucose reduces the viability of cortical astrocytes through increased permeability of connexin 43 hemichannels. Glia 58: 329-343.

73. Ginsberg MD (2008) Neuroprotection for ischemic stroke: past, present and future. Neuropharmacology 55: 363-389.

74. O'Collins VE, Macleod MR, Donnan GA, Horky LL, van der Worp BH, et al. (2006) 1,026 experimental treatments in acute stroke. Ann Neurol 59: 467-477.

75. Pandya RS, Mao L, Zhou H, Zhou S, Zeng J, et al. (2011) Central nervous system agents for ischemic stroke: neuroprotection mechanisms. Cent Nerv Syst Agents Med Chem 11: 81-97. 